\title{
Determination of the maximum effective burning velocity of dust-air mixtures in constant volume combustion
}

\author{
Y.K. Pu ${ }^{\mathrm{a}, *}$, F. Jia ${ }^{\mathrm{a}}$, S.F. Wang ${ }^{\mathrm{a}}$, T. Skjold ${ }^{\mathrm{b}, \mathrm{c}}$ \\ ${ }^{a}$ National Microgravity Laboratory, Institute of Mechanics, Chinese Academy of Sciences, Beijing 100080, China \\ ${ }^{\mathrm{b}}$ GexCon AS, Fantoftvegen 38, 5892 Bergen, Norway \\ ${ }^{\mathrm{c}}$ Department of Physics and Technology, University of Bergen, 5007 Bergen, Norway
}

Received 28 November 2006; received in revised form 18 April 2007; accepted 26 April 2007

\begin{abstract}
The reactivity of a combustible dust cloud is traditionally characterized by the so-called $K_{\mathrm{St}}$ value, defined as the maximum rate of pressure rise measured in constant volume explosion vessels, multiplied with the cube root of the vessel volume. The present paper explores the use of an alternative parameter, called the maximum effective burning velocity $\left(u_{\mathrm{eff}, \max }\right)$, which also is derived from pressure-time histories obtained in constant volume explosion experiments. The proposed parameter describes the reactivity of fuel-air mixtures as a function of the dispersion-induced turbulence intensity. Procedures for estimating $u_{\mathrm{eff}, \max }$ from tests in both spherical and cylindrical explosion vessels are outlined, and examples of calculated values for various fuel-air mixtures in closed vessels of different sizes and shapes are presented. Tested fuels include a mixture of $7.5 \%$ methane in air, and suspensions of $500 \mathrm{~g} / \mathrm{m}^{3} \mathrm{cornstarch}$ in air and $500 \mathrm{~g} / \mathrm{m}^{3}$ coal dust in air. Three different test vessels have been used: a $20-1$ spherical vessel and two cylindrical vessels, 7 and 221 . The results show that the estimated maximum effective burning velocities are less apparatus dependent than the corresponding $K_{\mathrm{St}}$ values. (C) 2007 Elsevier Ltd. All rights reserved.
\end{abstract}

Keywords: Dust explosion; Constant volume combustion; Maximum effective burning velocity

\section{Introduction}

In efforts to classify the relative reactivity of various combustible dusts, and thereby being able to predict the violence of accidental dust explosion in industrial plants, the US Bureau of Mines developed a series of closed bomb tests; vessel volumes ranging from 1.2 to 91 (Nagy \& Verakis, 1985). Two different 20-1 vessels were later developed, one in Europe (Siwek, 1977) and one in the US (Cashdollar \& Hertzberg, 1985); both are still widely used, since they are considered having the minimum practical volume for reliable determination of explosion indices for dust-air mixtures. In order to investigate dust explosions at scales closer to the ones met in industry, Bartknecht (1981) developed various larger explosion vessels $\left(1-10 \mathrm{~m}^{3}\right)$.

\footnotetext{
${ }^{*}$ Corresponding author. Tel.: + 861062615546 ; fax: + 861062561284 .

E-mail address: ykpu@imech.ac.cn (Y.K. Pu).
}

Explosion data obtained from closed vessel tests include the maximum pressure, $\Delta P_{\max }$, and the maximum rate of pressure rise, $(\mathrm{d} P / \mathrm{d} t)_{\max }$, representing the energy content and the reactivity of the combustible mixture, respectively. The $K_{\mathrm{St}}$ value, defined as $V_{0}^{1 / 3}(\mathrm{~d} P / \mathrm{d} t)_{\max }$, is in essence a scaled maximum rate of pressure rise based on the volume $V_{0}$ of the explosion vessel. Bartknecht (1981) introduced the so-called cubic law, stating that the $K_{\mathrm{St}}$ value should be a constant for a given dust sample. This assumption has later been used to extrapolate results obtained in laboratory scale test vessels to large-scale industrial enclosures. However, abundant experimental data have later shown that $K_{\mathrm{St}}$ values obtained from different test vessels may differ significantly for the same dust-air mixture, most recently demonstrated by Proust, Accorsi, and Dupont (2006). The difference is particularly pronounced for experiments performed in cylindrical or elongated vessels, which are quite common in industrial applications. Eckhoff (1977) demonstrated the influence of the ignition delay time on $K_{\mathrm{St}}$ values determined in the 1.2-1 Hartmann bomb. 
In his later publications (Eckhoff, 1984/1985, 1987) he pointed out that due to the total lack of account for the effect of dispersion-induced turbulence in the derivation of the cubic law, the $K_{\mathrm{St}}$ value is a parameter of inherent ambiguity. Nevertheless, it is still a common practice to report the reactivity of a combustible dust-air mixture as a $K_{\mathrm{St}}$ value, and this approach may to some degree be justified as long as the experiments are performed according to the specified procedures in standardized vessels (e.g. Cesana \& Siwek, 2001).

Dispersion systems are required for generating dust suspensions inside closed explosion vessels, and it is wellknown that the dispersion process is characterized by highly transient flow (Dahoe, Cant, \& Scarlett, 2001; Dahoe, van der Nat, Braithwaite, \& Scarlett, 2001; Dahoe, Zavenbergen, Lemkowitz, \& Scarlett, 1996; Pu, Li, Kauffman, \& Bernal, 1989). Since the decay of dispersioninduced turbulence is accompanied by deterioration in the actual dust concentration and agglomeration of dispersed particles, the maximum explosion pressure and maximum rate of pressure rise are not functions of turbulence alone. Nevertheless, it is important to characterize the features of the dispersion-induced turbulence by, e.g. decay laws for the root-mean-square of the turbulent velocity fluctuations $\left(u^{\prime}\right)$ and the turbulent integral length scale $\left(\ell_{e}\right)$ in these systems. A statistical method based on the ensemble average principle was adopted by $\mathrm{Pu}$ et al. (1989) in determining turbulence parameters for the dispersioninduced non-stationary flow in closed combustion vessels. More recent contributions include the ones by Dahoe et al. (1996), Dahoe, Cant et al. (2001), and Dahoe, van der Nat et al. (2001).

Through systematic experimental studies on dust combustion in closed vessels with different shape and volume $(\mathrm{Pu}, 1987 ; \mathrm{Pu}, \mathrm{Hu}$, \& Jia, 2000; Pu, Jarosinski, Johnson, \& Kauffman, 1990; Pu, Jarosinski, Tai, \& Kauffman, 1988; $\mathrm{Pu}$ et al., 1989; Pu, Yuan, Ding, \& Tang, 1993; Yan \& Pu, 1999) it has been possible to identify an alternative parameter, called the maximum effective burning velocity $\left(u_{\text {eff,max }}\right)$, that can be used to characterize the explosion characteristics of dust-air mixtures. The concept of maximum effective burning velocity was suggested by $\mathrm{Pu}$ et al. (1990), and a similar approach has recently been adopted for analyzing results from 20-1 explosion vessels as input to the CFD-code DESC (Skjold, 2006; Skjold, Arntzen, Hansen, Storvik, \& Eckhoff, 2006; Skjold et al., 2005). The main purpose of the present study is to investigate practical procedures for determining $u_{\mathrm{eff} \text { max }}$ from measured pressure-time histories in closed combustion vessels, and to compare results obtained in different explosion vessels. The approach followed is based on classical theory for constant volume explosions, and careful examination of the features of measured pressure-time traces, especially during the initial adiabatic stage of flame propagation in closed vessels. When both $K_{\mathrm{St}}$ and $u_{\text {eff,max }}$ values obtained in different vessels are plotted against the respective measured dispersion-induced turbulence intensities at time of ignition, the results show that $u_{\text {eff,max }}$ is less apparatus dependent than the $K_{\mathrm{St}}$ value.

\section{Theory}

\subsection{The validity of the cubic law}

The validity of the cubic law for dust-air mixtures can be examined by the classical theory for constant volume explosions (Bradley \& Mitcheson, 1976; Lewis \& von Elbe, 1987; Pu et al., 2000; Yan \& Pu, 1999). For the purpose of the present work, it is sufficient to just outline some essential features of this theory. First, we assume that the flame is thin compared with the characteristic length scale of the vessel. As shown by Dahoe et al. (1996), this assumption is not strictly valid for turbulent dust flames, but it will nevertheless be used as a first approximation. Second, it is assumed that the mixture behaves as a perfect gas, and that both the specific heat ratio $\gamma$ and the specific heat capacity at constant volume $C_{V}$ have average constant values for both burnt and unburnt mixture. Third, any measured pressure-time curve from constant volume combustion tests are regarded as the outcome of a propagating flame front progressively releasing energy, and thereby pressurizing the vessel. Under adiabatic conditions, the internal energy $(U)$ of a bulk mass of explosive mixture in a volume $\left(V_{0}\right)$ becomes:

$U=M C_{V} T=\frac{M R T}{\gamma-1}=\frac{P V_{0}}{\gamma-1}$,

where $T$ is the averaged temperature of the entire mass $(M)$ inside the volume. Thus, the rate of increase of internal energy in the volume becomes:

$\frac{\mathrm{d} U}{\mathrm{~d} t}=\frac{V_{0}}{\gamma-1} \frac{\mathrm{d} P}{\mathrm{~d} t}$.

Assuming the energy release to be caused by some fictitious (or real) propagating combustion front at a burning velocity $u(t)$ relative to the gas ahead of it, one can write:

$\frac{\mathrm{d} U}{\mathrm{~d} t}=\frac{V_{0}}{\gamma-1} \frac{\mathrm{d} P}{\mathrm{~d} t}=A(t) u(t) \rho(t) Q$,

where $Q$ is the specific chemical energy in the unburned mixture, and $\rho(t)$ and $A(t)$ are the gas density of the unburned mixture and the area of the flame front at time $t$, respectively. Assuming isentropic compression of the gas in the vessel, $\rho=\rho_{0}\left(P / P_{0}\right)^{1 / \gamma}$, and since $\rho_{0} Q=M Q / V_{0}$, we get:

$M Q=\Delta P_{\max } \frac{V}{\gamma-1}$,

where $\Delta P_{\max }$ is the maximum pressure increase during the whole combustion process. Combining Eqs. (3) and (4) yields:

$\frac{V_{0}}{A(t)} \frac{\mathrm{d} P}{\mathrm{~d} t}=u(t) \Delta P_{\max }\left(\frac{P}{P_{0}}\right)^{1 / \gamma}$. 
At the inflection point of the pressure-time curve, i.e. when the rate of pressure rise reaches its maximum value $(\mathrm{d} P / \mathrm{d} t)_{\max }$ at time $t^{*}$, Eq. (5) becomes:

$\frac{V_{0}}{A\left(t^{*}\right)}\left(\frac{\mathrm{d} P}{\mathrm{~d} t}\right)_{\max }=u_{\max } \Delta P_{\max }\left(\frac{P\left(t^{*}\right)}{P_{0}}\right)^{1 / \gamma}$.

Since the area and volume of a spherical flame with radius $r$ is $A(t)=4 \pi r^{2}$ and $V(t)=4 \pi r^{3} / 3$, respectively, the flame area may be expressed as $A(t)=C_{\mathrm{B}} V(t)^{2 / 3}$ where $C_{\mathrm{B}}\left(=4(3 / 4)^{2 / 3} \pi^{1 / 3}\right)$ is a constant of proportionality. The empirical correlation $r\left(t^{*}\right) \sim 0.8 R$ has been suggested for centrally ignited dust explosions in a closed spherical vessel with radius $R$ (Kauffman, Srinath, Tezok, Nicholls, \& Sichel, 1984; Pu et al., 1990; Pu et al., 1988). Hence, $V_{0} / A\left(t^{*}\right)=V_{0}^{1 / 3} / C_{\mathrm{A}}, C_{\mathrm{A}} \approx(0.8)^{2} C_{\mathrm{B}}$, and Eq. (6) becomes:

$K_{\mathrm{St}}=\left(\frac{\mathrm{d} P}{\mathrm{~d} t}\right)_{\max } V_{0}^{1 / 3}=C_{\mathrm{A}} u_{\max } \Delta P_{\max }\left(\frac{P\left(t^{*}\right)}{P_{0}}\right)^{1 / \gamma}$.

Furthermore, measured pressure-time traces $(\mathrm{Pu}$ et al., $1988,1990)$ indicate that $\Delta P\left(t^{*}\right) \approx 0.5 \Delta P_{\max }$. Hence, the value of the $K_{\mathrm{St}}$ factor in Eq. (7) may be approximated by the three parameters $C_{\mathrm{A}}, u_{\max }$ and $\Delta P_{\max }$, and the validity of cubic law is limited to flame propagating in a spherical vessel with center ignition (i.e. $C_{\mathrm{A}}=$ constant), to the laminar case (i.e. $u_{\max }=u_{l}=$ constant), and to a given dust mixture (i.e. $\Delta P_{\max }=$ constant). Therefore, it seems reasonable to assume that the non-spherical shape of many test vessels, and the transient nature of the dispersion-induced turbulent flows, introduce severe limitations to the applicability of the cubic law.

\subsection{The transient nature of dispersion-induced turbulence}

It is well known that both the design of the dust dispersion system, and the ignition delay time used, influence the explosion development in closed vessels significantly (Dahoe, Cant et al., 2001; Dahoe, van der Nat et al., 2001; Pu, 1987). Pu et al. developed dust dispersion systems with single and double rows of small jets producing small-scale turbulence (Pu, 1987; $\mathrm{Pu}$ et al., 2000; Pu et al., 1993; Yan \& Pu, 1999). Pu et al. (1989) measured the transient nature of the dispersion-induced turbulence in a 20-1 spherical vessel and 22-1 cylindrical vessels with hot wire anemometry, and estimated $u^{\prime}$ by an ensemble average method. Yan and $\mathrm{Pu}(1999)$ measured the transient decay of dispersion-induced turbulence from a two-row jets dispersion system in a 7-1 cylindrical vessel. Fig. 1 summarizes the results for the various vessels. The results from the 20-1 closed spherical vessel in Fig. 1a show that the instantaneous turbulent RMS velocities reach maximum values of about $3 \mathrm{~m} / \mathrm{s}$ after a very short time $(20-50 \mathrm{~ms})$, then decay to about $1.5 \mathrm{~m} / \mathrm{s}$ after $100 \mathrm{~ms}$, to $0.5 \mathrm{~m} / \mathrm{s}$ after $200 \mathrm{~ms}$, and finally to $0.25 \mathrm{~m} / \mathrm{s}$ after $400 \mathrm{~ms}$. At the same time, the mean value of the integral length scale increases from about 10 to about $15 \mathrm{~mm}$ after $300 \mathrm{~ms}$, and to about $25 \mathrm{~mm}$ after $600 \mathrm{~ms}$. The results from
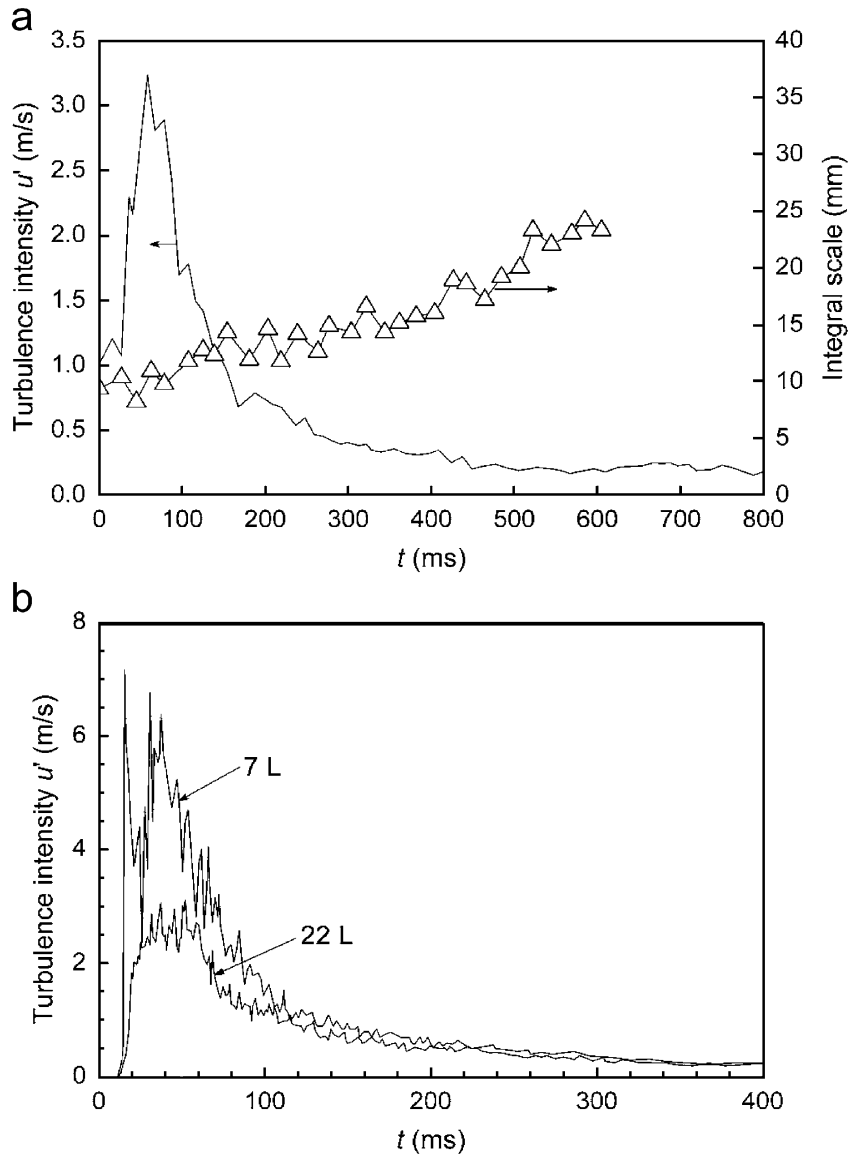

Fig. 1. Ensemble average RMS turbulent velocities/integral scales induced by dispersion system as a function of time in closed vessels. (a) In 201 spherical vessel (Pu et al., 1990); (b) in two cylindrical vessels, 7 and 221 (Yan \& Pu, 1999).

the 22 and 71 closed cylindrical vessels in Fig. $1 \mathrm{~b}$ indicate that the instantaneous turbulent RMS velocities reach their maximum values of 2.5 and $5.5 \mathrm{~m} / \mathrm{s}$, respectively, after $20-50 \mathrm{~ms}$, then decay to 2.0 and to $1.5 \mathrm{~m} / \mathrm{s}$, respectively, after $100 \mathrm{~ms}$, to $0.5 \mathrm{~m} / \mathrm{s}$ in both vessels after $200 \mathrm{~ms}$, and finally to $0.25 \mathrm{~m} / \mathrm{s}$ in both vessels after $400 \mathrm{~ms}$. Empirical decay laws extracted from such measurements can be used to estimate turbulence intensity and integral length scale as a function of time. Hence, the turbulence intensity at time of ignition, $u^{\prime}\left(t_{\mathrm{ig}}\right)$, can replace the ignition delay time, $t_{\mathrm{v}}$, as both independent variable and reference point for measurements obtained in different experiments. There are, however, several sources of uncertainty associated with measurements in transient particle-laden flows. It is not straightforward to unambiguously define an average velocity in a transient flow field, and hence to obtain well-defined values for the root-mean-square of the turbulent velocity fluctuations (Dahoe, Cant et al., 2001; Dahoe, van der Nat et al., 2001; Skjold, 2003), and certain assumptions must be invoked in order to extract decay laws for the turbulent integral length scale from such measurements (Dahoe, van der Nat et al., 2001; Pu et al., 1989). Furthermore, the flow measurements must be 
performed with a very limited amount of dust in the system, and the influence of the presence of dust particles on the flow is usually unknown (Dahoe, van der Nat et al., 2001; Skjold, 2003). Finally, by using the level of turbulence at time of ignition as a reference, any decay of turbulence during the period from $t_{\mathrm{ig}}$ to $t^{*}$ is neglected; an alternative approach involves using the level of turbulence at the inflection point as reference, hence neglecting any turbulence induced by combustion during the same period (Skjold, 2006; Skjold et al., 2005, 2006).

\subsection{The maximum effective burning velocity}

The effective burning velocity introduced here is a conceptual quantity; it is the burning velocity of an idealized flame, equivalent to the real dust flame, in the sense that both have similar pressure-time traces. Hence, $u_{\text {eff,max }}$ is not necessarily the true burning velocity, and the present approach can only be justified by its improved performance in correlating experimental data from vessels of varying size and shape. Nevertheless, assuming that the energy release in the dust combustion process is equivalent to some fictitious propagating flame front at a burning velocity $u_{\text {eff }}(t)$ relative to the unburnt mixture, Eq. (5) becomes:

$\frac{V_{0}}{A(t)} \frac{\mathrm{d} P}{\mathrm{~d} t}=u_{\mathrm{eff}}(t) \Delta P_{\max }\left(\frac{P}{P_{0}}\right)^{1 / \gamma}$,

and $u_{\text {eff }}(t)$ can be represented as

$u_{\text {eff }}=\frac{P_{0}^{1 / \gamma}}{\Delta P_{\max }} \frac{V_{0}}{A(t)} \frac{1}{P^{1 / \gamma}} \frac{\mathrm{d} P}{\mathrm{~d} t}$.
Hence, as we approach the inflection point: $t \rightarrow t^{*}, \mathrm{~d} P / \mathrm{d} t \rightarrow$ $(\mathrm{d} P / \mathrm{d} t)_{\max }$ and $u_{\text {eff }}(t) \rightarrow u_{\text {eff,max }}$, i.e.:

$u_{\text {eff } \max }=\frac{P_{0}^{1 / \gamma}}{\Delta P_{\max }} \frac{V_{0}}{A\left(t^{*}\right)} \frac{1}{P\left(t^{*}\right)^{1 / \gamma}}\left(\frac{\mathrm{d} P}{\mathrm{~d} t}\right)_{\max }$

Eq. (10) is the general expression for the maximum effective burning velocity used in the present study. The volume and shape of the test vessel are represented by the ratio $V_{0} / A\left(t^{*}\right)$. Quantitative estimation of $u_{\text {eff,max }}$ requires careful examination of measured pressure development and flame propagation in the constant volume test vessel to deal with the following complex issues: (1) how to include the influence of the transient dispersion induced turbulence; and, (2) how to estimate the ratio of vessel volume to flame area, i.e. $V_{0} / A\left(t^{*}\right)$.

\subsection{Combustion processes in spherical and cylindrical closed vessels}

Visual observations by Ellis (1928) and $\mathrm{Hu}, \mathrm{Pu}, \mathrm{Jia}$, and Jarosinski (2005), as well as pressure-time traces ( $\mathrm{Pu}, 1987$; $\mathrm{Pu}$ et al., 2000, 1990, 1988, 1993) and direct wall heat transfer measurements $(\mathrm{Pu}, 1987)$, suggest that combustion process in closed vessels can be divided into two subprocesses: an initial adiabatic process, followed by a nonadiabatic process. The thermodynamic analysis used to determine the value of the maximum effective burning velocity by Eq. (10) applies only to the first adiabatic stage of constant volume combustion, during which the flame surface remains simple and regular in shape.

After central ignition in a closed spherical vessel, the flame front propagates in radial direction, keeping its spherical shape and symmetry, until almost touching the
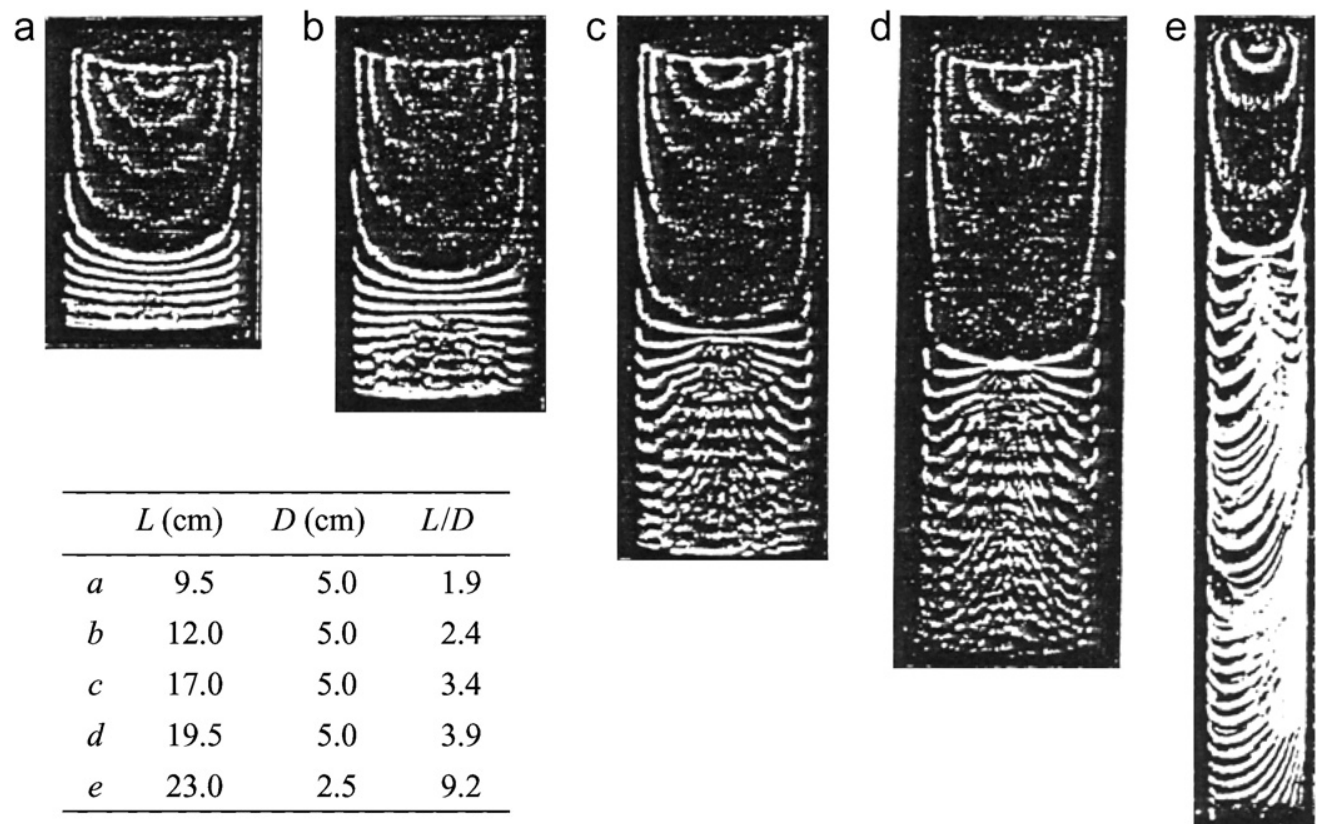

Fig. 2. Tracing of flame front propagating inside a closed tube in mixture of 10 parts $\mathrm{CO}$ and 1 part $\mathrm{O}_{2}$, from Ellis (1928). 
wall. One can usually regard this initial stage of constant volume spherical combustion as adiabatic. However, as the flame approaches the wall, heat loss is no longer negligible, and the flame is distorted.

For constant volume combustion in cylindrical vessels, with bottom-end ignition, the flame front expands both in radial and axial directions. The initially hemispherical flame front evolves into an increasingly elongated ellipsoidal surface. Due to heat loss to the wall the surface of the flame front shrinks and becomes flattened, then wrinkled. In the final stage of flame propagation the wellknown unsteady tulip flame dominates. Although the entire process involves some heat loss to the wall, we nevertheless regard the initial phase of constant volume combustion in cylindrical closed vessels as approximately adiabatic. Ellis (1928) investigated and visualized gas flames in mixture of 10 parts $\mathrm{CO}$ and 1 part $\mathrm{O}_{2}$, propagating in closed cylindrical vessels of aspect ratios from 1 to 10 (see Fig. 2). From Fig. 2, it is possible to estimate the distance $L_{\text {eff }}$ at which the ellipsoidal flame surface becomes flattened. Fig. 3 shows the relationship between the aspect ratio of a cylindrical vessel and the value of the corresponding $L_{\text {eff }}$ obtained by Ellis (1928),

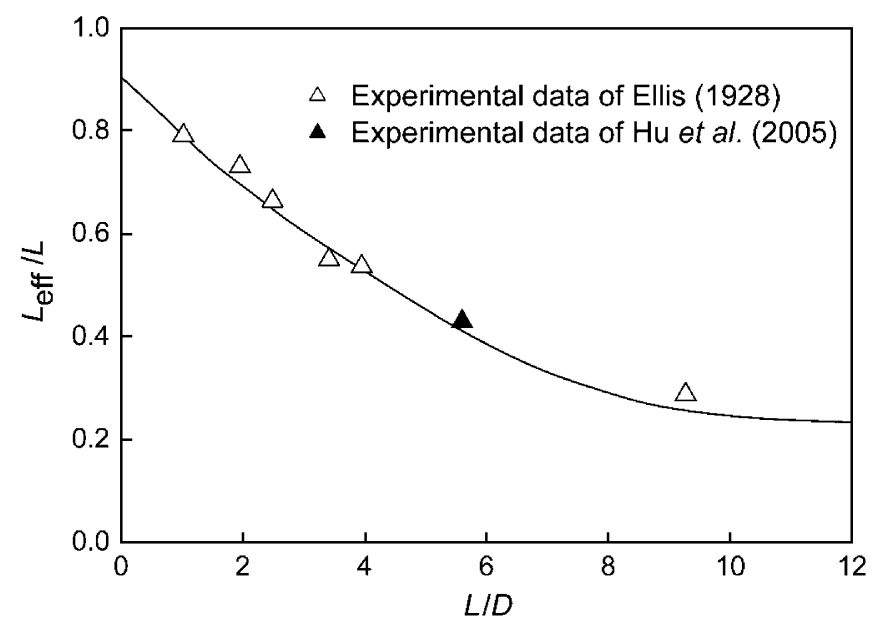

Fig. 3. An empirical correlation from Ellis (1928) between the ratio of $L / D$ and measured data of $L_{\mathrm{eff}} / D$ in closed combustion cylindrical vessels.

and one experimental point obtained by $\mathrm{Hu}$ et al. (2005). Fig. 4 shows experimental results obtained by $\mathrm{Hu}$ et al.: flame position and shape as a function of time for $4.1 \%$ propane-air mixture in a closed cylindrical tube $(L=1 \mathrm{~m}$, $D=0.18 \mathrm{~m}, L / D=5.6$ ).

\subsection{Estimating the ratio of vessel volume to flame area}

The value of $V_{0} / A\left(t^{*}\right)$ must be estimated in order to obtain the maximum effective burning velocity from Eq. (10). Let $t^{* *}$ denote the time after ignition at which the first stage of combustion ends. Hence, for a spherical vessel, while $t<t^{* *}$, we have:

$\frac{V_{0}}{A(t)}=\frac{4 / 3 \pi R_{0}^{3}}{4 \pi R(t)^{2}}=\frac{1}{3} \frac{R_{0}^{3}}{R(t)^{2}}$.

Measured pressure-time traces for both gas and dust explosions in spherical vessels (Kauffman et al., 1984; $\mathrm{Pu}$ et al., 2000) indicate that the flame radius $r\left(t^{* *}\right)$ is approximately 0.8 times the radius $R_{0}$ of test vessel as $t \rightarrow t^{* *}$, hence:

$\frac{V_{0}}{A\left(t^{* *}\right)} \approx 0.52 R_{0}$.

For a cylindrical vessel it seems reasonable to assume a semi-ellipsoidal flame front during the first stage of combustion, hence:

$\frac{V_{0}}{A(t)}=\frac{\pi(D / 2)^{2} L}{\pi d(t)\left(\left(7 l(t)^{2}+6 l(t) d(t)-d(t)^{2}\right) /(6 l(t))\right)}$,

where $l(t)$ and $d(t)$ are the half lengths of the long and short axis, respectively, of the ellipsoidal flame surface and $D$ and $L$ are the diameter and the length of the vessel, respectively. As $t \rightarrow t^{* *}, l\left(t^{* *}\right) \rightarrow L_{\text {eff }}$ and $d\left(t^{* *}\right) \rightarrow D$; hence Eq. (13) can be written as

$\frac{V_{0}}{A\left(t^{* *}\right)}=\frac{\pi(D / 2)^{2} L}{\pi D\left(7 L_{\mathrm{eff}}^{2}+6 L_{\mathrm{eff}} D-D^{2} / 6 L_{\mathrm{eff}}\right)}$.

Eq. (14) has been used to estimate the ratio of vessel volume to flame area for cylindrical vessels in the present work.

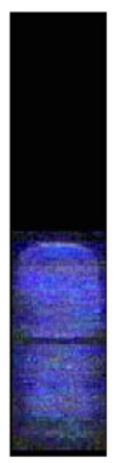

60

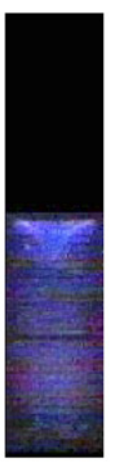

76

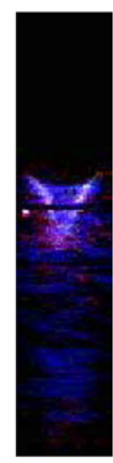

104

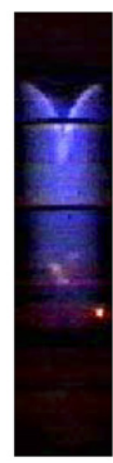

168

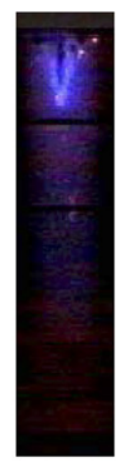

204

Fig. 4. Flame position and its shape as a function of time. Combustion of $4.1 \% \mathrm{C}_{3} \mathrm{H}_{8}$-air mixture in a closed tube $(L=1 \mathrm{~m}, D=0.18 \mathrm{~m}, L / D=5.6)$ (Hu et al., 2005). The flame contacts with the vessel sidewalls at $t=52 \mathrm{~ms}$. 


\subsection{Estimating the maximum effective burning velocity}

Measured pressure-time traces from a large number of experiments carried out in both spherical and cylindrical closed vessels, involving both gaseous fuels and dust-air mixtures (Pu, 1987; Pu et al., 2000, 1990, 1988, 1993), suggest that the inflection point $\left(t \rightarrow t^{*}\right)$ represents the transition from the initial adiabatic stage to the subsequent non-adiabatic stage. Hence, we further assume that $t^{* *}$ equals $t^{*}$, i.e. that the rate of pressure rise, $\mathrm{d} P / \mathrm{d} t$, and the corresponding effective burning velocity, $u_{\mathrm{eff}}(t)$, reach their maximum values, $(\mathrm{d} P / \mathrm{d} t)_{\max }$ and $u_{\mathrm{eff}, \max }$ at the same instant. After identifying the inflection point, i.e. $t^{*}$, $P\left(t^{*}\right), \Delta P_{\max }$ and $(\mathrm{d} P / \mathrm{d} t)_{\max }$, from a measured pressure-time trace, the parameter $V_{0} / A\left(t^{*}\right)\left(=V_{0} / A\left(t^{* *}\right)\right)$ can be estimated from Eq. (12) for spherical vessels or from Eq. (14) for cylindrical vessels, and it is straightforward to estimate the value of maximum effective burning velocity, $u_{\text {eff,max }}$, at $t^{*}$ from Eq. (10). Furthermore, if the dispersioninduced turbulence can be determined, the maximum efficient burning velocity can be reported as a function of turbulence intensity.

\section{Results}

The applicability of the maximum effective burning velocity as a parameter describing the reactivity of fuel-air mixtures was investigated for constant volume combustion data obtained in four explosion vessels of various shapes and sizes; the fuels included cornstarch, coal dust, and methane gas.

Since the dispersion induced flow was measured in all studies, the influence of turbulence intensity on the parameters $u_{\text {eff,max }}$ and $K_{\mathrm{St}}$ can be studied. Fig. 5 illustrates the correlation of the maximum effective burning velocities with dispersion-induced turbulence intensity at the time of ignition for various fuel-air mixtures.

For $7.5 \%$ methane in air (Fig. 5a) and $500 \mathrm{~g} / \mathrm{m}^{3}$ cornstarch in air (Fig. 5b), the pressure-time traces were measured in vessels of different shape (spherical and cylindrical) but similar volume (20 and 221). For the $500 \mathrm{~g} / \mathrm{m}^{3}$ coal-air mixture (Fig. $5 \mathrm{c}$ ), the pressure-time traces were measured in vessels with different shapes (spherical and cylindrical) but similar volume (20 and 221 ), and similar shape (cylindrical) but different volumes (7 and 221).

Although the maximum effective burning velocities in Fig. 5 originate from different vessels, a near linear correlation is observed between the maximum effective burning velocity and the turbulence intensity at the time of ignition. The results resemble those of previous investigations (Chomiak \& Jarosinski, 1982) and recent experiments (Hu et al., 2005) for gaseous fuel-air mixture with smallscale turbulence.

Fig. 6 illustrates correlation of the corresponding $K_{\mathrm{St}}$ values with dispersion-induced turbulence intensity at time of ignition. It is evident that quite different $K_{\mathrm{St}}$ values may
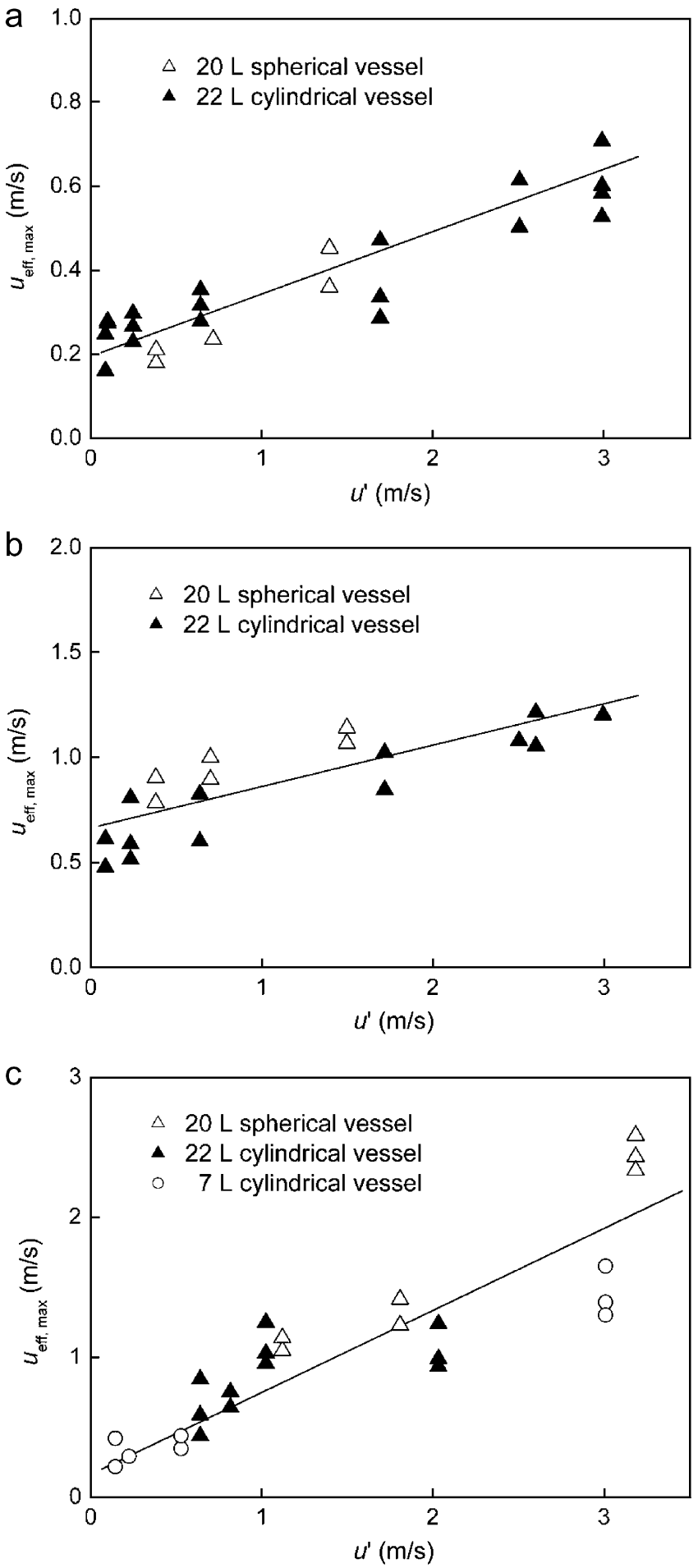

Fig. 5. The maximum effective burning velocity as a function of RMS turbulent velocities induced by dispersion system in three testing vessels. (a) For 7.5\% $\mathrm{CH}_{4}$-air mixtures; (b) for cornstarch dust-air mixtures (dust concentration $500 \mathrm{~g} / \mathrm{m}^{3}$ ); and (c) for coal dust-air mixtures (dust concentration $500 \mathrm{~g} / \mathrm{m}^{3}$ ).

be obtained for the same fuel-air mixture when it is tested in different test vessels, and with different dust dispersion systems. 

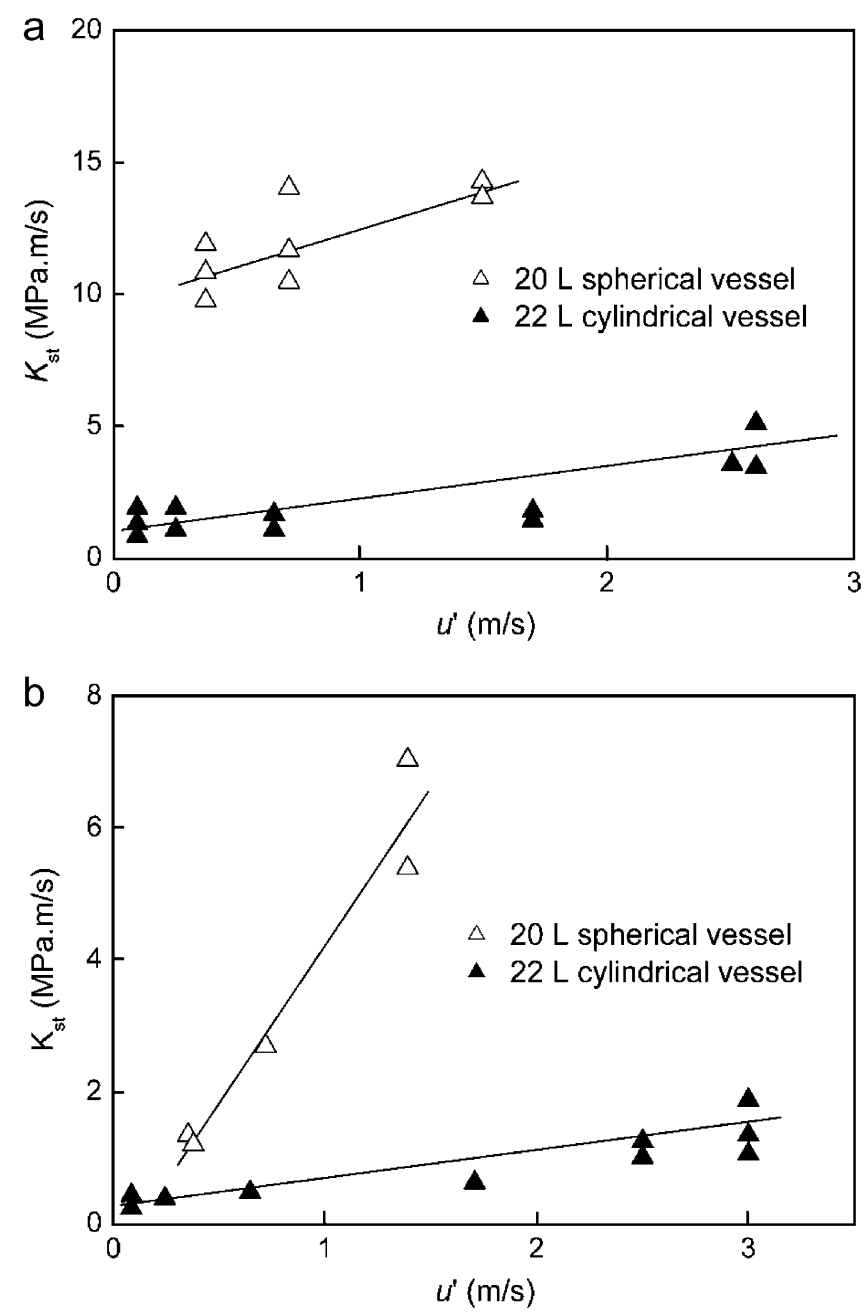

Fig. 6. The values of $K_{\mathrm{St}}$ as a function of RMS turbulent velocities induced by dispersion system in testing vessels of 201 sphere and 221 cylinder. (a) For 7.5\% $\mathrm{CH}_{4}$-air mixtures and (b) for cornstarch dust-air mixtures (dust concentration $500 \mathrm{~g} / \mathrm{m}^{3}$ ).

\section{Discussion and conclusions}

The maximum effective burning velocity $\left(u_{\text {eff,max }}\right)$ has been suggested as an alternative, or additional, parameter to the traditional $K_{\mathrm{St}}$ value. A comparison of Figs. 5 and 6 suggests that $u_{\text {eff,max }}$ is less apparatus dependent than the $K_{\mathrm{St}}$ value; this is primarily a result of the fact that the turbulent flow conditions in the mixture are taken into account. Although the main results can be derived from classical theory for constant volume combustion, some of the assumptions and empirical relations, e.g. $r\left(t^{*}\right) \approx 0.8 R_{0}$, $\Delta P\left(t^{*}\right) \approx 0.5 \Delta P_{\max }$, and $t^{*} \approx t^{* *}$, originate from a limited number of experimental studies of constant volume combustion. Hence, the proposed parameter is essentially of empirical nature, and the approach adopted in the present work cannot be expected to perform equally well for all vessel arrangements and fuel-air mixtures; weakly reacting mixtures and small vessel volumes should definitely be avoided.
The turbulence measurements referred to in this paper were obtained by hot wire anemometry, and since this method inevitably interferes with the flow field, there will be some discrepancies between these data and data obtained by, e.g. laser Doppler anemometry. The dispersion system, including the volume and initial pressure in the reservoir, the type of dispersion nozzle used, the opening time and cross section of the valves, etc., will also influence on the results. Finally, the results may depend on the theoretical assumptions and numerical methods used when analyzing and processing the experimental data. It is not straightforward to measure turbulence parameters, such as RMS velocity and integral length scale, in highly transient flow fields, and future work is required in this area.

When studying real dust flames in closed vessels, several complicating factors should be taken into account. The structure of a dust flame is rather complex, and, for organic materials, two distinct flame regions have been suggested: a thin region characterized by homogeneous combustion of volatiles, and a tail region characterized by heterogeneous combustion of particles (Jarosinski, Pu, Bulewicz, Kauffman, \& Johnson, 1993; Mazurkiewicz, Jarosinski, \& Wolanski, 1993; $\mathrm{Pu}, 1987)$. The relative contribution to the total energy release, and hence the measured rate of pressure rise, from combustion of volatiles is not easy to estimate; it is probably determined by such factors as particle size distribution and volatile content of the particles - finer particles and higher volatile content favoring increased release of energy in the flame front.

The maximum effective burning velocity is a conceptual quantity, and should not be interpreted as an actual turbulent burning velocity $\left(u_{\mathrm{t}}\right)$. However, it has the same dimensions as burning velocity, and future investigations may reveal correlations between $u_{\text {eff,max }}$ and $u_{\mathrm{t}}$. If reliable correlations between the turbulence parameters $u^{\prime}$ and $\ell_{e}$, and turbulent burning velocities in dust clouds, can be extracted from constant volume explosion vessels, it will be of great value when modeling industrial dust explosions by analytical methods, computational fluid dynamics, or other means.

\section{Acknowledgment}

The work reported here was sponsored by the Knowledge Innovation Major Project of Chinese Academy of Sciences under Grant number KJCX2-SW-L05.

\section{References}

Bartknecht, W. (1981). Explosions: Course, prevention, and protection New York: Springer.

Bradley, D., \& Mitcheson, A. (1976). Mathematical solutions for explosions in spherical vessel. Combustion and Flame, 26, 201-217.

Cashdollar, K. L., \& Hertzberg, M. (1985). 20-1 explosibility test chamber for dusts and gases. Review of Scientific Instruments, 56(4), 596-602.

Cesana, C., \& Siwek, R. (2001). Operating instructions 20-l-apparatus, 6.0. CH-4127 Birsfelden, Switzerland: Adolf Kühner AG. 
Chomiak, J., \& Jarosinski, J. (1982). Flame quenching by turbulence. Combustion and Flame, 48, 241-249.

Dahoe, A. E., Cant, R. S., \& Scarlett, B. (2001). On the decay of turbulence in the 20-litre explosion sphere. Flow, Turbulence and Combustion, 67, 159-184.

Dahoe, A. E., van der Nat, K., Braithwaite, M., \& Scarlett, B. (2001). On the sensitivity of the maximum explosion pressure of a dust deflagration to turbulence. KONA, 19, 178-195.

Dahoe, A. E., Zavenbergen, J. F., Lemkowitz, S. M., \& Scarlett, B. (1996). Dust explosions in spherical vessels: The role of flame thickness in the validity of the 'cube-root law'. Journal of Loss Prevention in the Process Industries, 9, 33-44.

Eckhoff, R. K. (1977). The use of the Hartmann bomb for determining $K_{\mathrm{St}}$ values of explosible dust clouds. Staub-Reinhaltung der Luft, 37, $110-112$.

Eckhoff, R. K. (1984/1985). Use of $(\mathrm{d} P / \mathrm{d} t)_{\max }$ from closed bomb-dust tests for predicting violence of accidental dust explosions in industrial plants. Fire Safety Journal, 8, 159-168.

Eckhoff, R. K. (1987). Measurement of explosion violence of dust clouds. In Proceeding of International Symposium on Explosion Hazard Classification of Vapors, Gases and Dusts. Washington DC: National Academy Press Publication NMAB-447.

Ellis, C. (1928). Flame movement in gaseous explosive mixtures. Fuel in Science and Practice, 7, 502-508.

Hu, J., Pu, Y. K., Jia, F., \& Jarosinski, J. (2005). Study on explosion venting in cylindrical vessel. Combustion Science and Technology, 177(2), 323-346.

Jarosinski, J., Pu, Y. K., Bulewicz, E. M., Kauffman, C. W., \& Johnson, V. G. (1993). Some fundamental characteristics of cornstarch dust-air flames. Progress in Astronautics and Aeronautics, 152, 119-135.

Kauffman, C. W., Srinath, S. R., Tezok, F. I., Nicholls, J. A., \& Sichel, M. (1984). Turbulent and accelerating dust flames. In 20th symposium (Int.) on combustion (pp. 1701-1708). The Combustion Institute.

Lewis, B., \& von Elbe, G. (1987). Combustion, flames and explosions of gases (3rd ed.). Orlando: Academic Press.

Mazurkiewicz, J., Jarosinski, J., \& Wolanski, P. (1993). Investigations of burning properties of cornstarch dust-air flames. Archivum Combustionis, 13, 189-201.

Nagy, J., \& Verakis, C. (1985). Development and control of dust explosions. New York: Marcel Dekker.

Proust, Ch., Accorsi, A., \& Dupont, L. (2006). Measuring the violence of dust explosion with the "20 liter sphere" and with the standard "ISO $1 \mathrm{~m}^{3}$ vessel". In Proceedings of the 6th international symposium on hazards, prevention, and mitigation of industrial explosions (Vol. II, pp. 647-661), August 27-September 1, Dalhousie University, Halifax, Nova Scotia, Canada.

$\mathrm{Pu}, \mathrm{Y} . \mathrm{K}$. (1987). Fundamental characteristics of laminar and turbulent flames in cornstarch dust-air mixtures. Ph.D. thesis. McGill University, Montreal.

Pu, Y. K., Hu, J., \& Jia, F. (2000). Systematic experimental studies on explosion characteristics for fine soft coal dust-air mixtures. Explosion and Shock Waves (in Chinese), 20(4), 303-312.

Pu, Y. K., Jarosinski, J., Johnson, V. G., \& Kauffman, C. W. (1990). Turbulence effects on dust explosions in the 20-liter spherical vessel. In 23rd symposium (int.) on combustion (pp. 843-849). The Combustion Institute.

Pu, Y. K., Jarosinski, J., Tai, C., \& Kauffman, C. W. (1988). The investigation of the effects of dispersion induced turbulence and its effects on dust explosion in closed vessel. In 22nd symposium (int.) on combustion (pp. 1777-1787). The Combustion Institute.

Pu, Y. K., Li, Y. C., Kauffman, C. W., \& Bernal, L. P. (1989). The determination of turbulence parameters in closed explosion vessels. AIAA Progress in Astronautics and Aeronautics, 132, 107-123.

Pu, Y. K., Yuan, S. X., Ding, D. Y., \& Tang, M. J. (1993). Systematic experimental studies on explosion characteristics for fine aluminum dust-air mixtures. Explosion and Shock Waves (in Chinese), 13(3), 192-204.

Siwek, R. (1977). 20-L Laborapparatur für die Bestimmung der Explosionskenngrößen brennbarer Stäube. Thesis (in German). HTL Winterthur, Switzerland.

Skjold, T. (2003). Selected aspects of turbulence and combustion in 20-litre explosion vessels. Cand. Scient. Thesis. Department of Physics, University of Bergen, Norway. Available (2007, March 15): 〈http:// www.ub.uib.no/elpub/2003/h/404002/ $>$.

Skjold, T. (2006). Review of the DESC project. In Proceedings of the sixth international symposium on hazards, prevention, and mitigation of industrial explosions (Vol. I, pp. 1-21), August 27-September 1, Dalhousie University, Halifax, Nova Scotia, Canada.

Skjold, T., Arntzen, B. J., Hansen, O. J., Storvik, I. E., \& Eckhoff, R. K. (2006). Simulation of dust explosions in complex geometries with experimental input from standardized tests. Journal of Loss Prevention in the Process Industries, 19, 210-217.

Skjold, T., Arntzen, B. J., Hansen, O. R., Taraldset, O. J., Storvik, I. E., \& Eckhoff, R. K. (2005). Simulating dust explosions with the first version of DESC. Process Safety and Environmental Protection, 83(B2), $151-160$.

Yan, N., \& Pu, Y. K. (1999). The determination of the dispersion induced turbulence in closed cylindrical explosion vessels. Journal of Experimental in Fluid Mechanics (in Chinese), 13(3), 59-64. 